\title{
Improving handovers across a North London Mental Health Trust
}

Jennifer Perry, Reena Manghnani, Andrew Sommerlad, George Ikkos

Barnet, Enfield and Haringey Mental Health Trust

\begin{abstract}
The GMC Survey June 2011 highlighted that trainees in Barnet, Enfield and Haringey Mental Health Trust (BEH-MHT) were dissatisfied with the Trust's handover process. At this time there were no Trust guidelines on handovers.
\end{abstract}

A quality improvement project was developed to create a consistent out of hours handover process and to improve staff satisfaction thereafter. Handover guidelines were implemented throughout the Trust on 1/2/12. An audit was carried out to measure staff compliance with handovers. A questionnaire was sent out to staff before and after implementation of the guidelines to assess satisfaction with handovers.

The audit results highlighted that handovers occurred relatively consistently across the Trust following implementation of the guidelines. The questionnaire results showed an improvement in staff satisfaction with the handover process.

The clinical implication of this project is an improved process of out of hours handover across BEH-MHT.

\section{Problem}

Barnet, Enfield and Haringey Mental Health Trust (BEH-MHT) provides a variety of mental health services across three boroughs in north London. There are three main hospital sites; St Ann's (Haringey), Chase Farm (Enfield) and Barnet+Edgware (Barnet). BEH-MHT provides training posts for Psychiatry and General Practice junior doctors who rotate every six months through different placements. The General Medical Council (GMC) conducts an Annual National Survey of Trainees and the June 2011 Survey showed, in response to questions about 'handover arrangements before/after night duty', that trainees were dissatisfied with handover arrangements within the Trust (1). This score represented the lowest score amongst all London mental health trusts.

In August 2011, after this survey was conducted, BEH-MHT introduced a new model for the provision of out-of-hours services, namely 'Mental Health at Night'. This resulted in the on-call junior doctor being non-residential and the first-line of emergency duty overnight being performed by a Senior Nurse Assessor (SNA). The SNA was able to contact a number of other doctors if medical assistance was required overnight. It was acknowledged that this would result in additional multidisciplinary handovers and lead to fewer face-to-face handovers. This more fragmented out-of-hours service could create greater dissatisfaction with provision of handover amongst trainees. At this time there were no Trust guidelines outlining the process of handover.

A quality improvement project was developed with support from the Trust's Medical Education Committee. It was carried out under the guidance of the London Deanery STeLI (Simulation and Technology-enhanced Learning Initiative) programme (2). The aims of the project were;

1. To develop an effective and consistent process for out-of hours handover,

2. To improve satisfaction with the handover process within the Trust.

At the time 'Mental Health at Night' was introduced, the out-of-hours on-call system was highly complex and varied from site to site throughout BEH-MHT. At St Ann's Hospital the on-call doctors worked a 24 hour shift (9am-9am). At Chase Farm Hospital and Barnet+Edgware Hospital the on-call doctors worked a 16 hour shift (5pm-9am) during the weekdays and a 24 hour shift at weekends. At these two hospital sites the weekday daytime shift from $9 \mathrm{am}-5 \mathrm{pm}$ would be covered by two different doctors; one working the morning $(9-1 \mathrm{pm})$ shift and the other working the afternoon (1-5pm) shift.

At all three hospital sites the doctor would be the first point of call up until 9pm and their on-calls were non-residential (except at Chase Farm where doctors carried a bleep and were residential 9am-9pm and non-residential thereafter). From 9pm-9am an SNA at each site would be the first point of call. If the SNA required medical assistance overnight they were able to contact a 'voluntary' doctor who covered all three sites from 10pm-8am. Between the hours of $9 \mathrm{pm}-10 \mathrm{pm}$ and $8 \mathrm{am}-9 \mathrm{am}$, if the SNA required medical assistance, they could contact the non-residential junior doctor oncall for their respective hospital sites. The SNA was also able to contact the on-call specialist registrar overnight if required. As a result the on-call junior doctor would rarely be contacted between the hours of $10 \mathrm{pm}-8 \mathrm{am}$ as the SNA, voluntary doctor, and specialist registrar should provide cover over this period.

At St Ann's and Barnet+Edgware hospitals the junior doctors and SNAs used mobile phones as a method of communication whilst oncall. At Chase Farm Hospital a bleep system was used instead; this hospital was unable to change to a mobile phone system as would be preferred for 'Mental Health at Night'. Doctors and SNAs on-call 
covered the inpatient wards and the Acute Assessment Centre (Chase Farm and St Ann's only) at their respective hospital sites.

\section{Background}

Within healthcare, 'handover' is the episode in which the care of, and responsibility for, a patient is transferred from one professional to another. When considering the safety of healthcare, handovers have attracted increasing attention from organisations such as the World Health Organisation (3) and the British Medical Association (4).

There is a developing evidence base (5) to suggest that clinicians are frequently dissatisfied with the provision of adequate time for handovers and quality of the information provided within this time (6). Although studies to date have not been able to robustly establish the effect of handover interventions on patient safety (7), it is thought that inadequate handover can result in numerous adverse effects. Impaired communication during handovers may lead to delays in diagnosis or treatment, resulting in potential adverse events or near misses $(8,9)$. It is thought that deficient handovers contribute to around one quarter of medical negligence claims $(10,11)$.

Within mental healthcare, the available literature focuses mainly on nursing handovers $(12,13)$. However, a recent observational study of handovers in mental health identified that Psychiatrists were less likely than other mental health professionals to have a face-to-face handover and were more likely to believe that shift-changes were problematic for patients (14).

\section{Baseline Measurement}

In December 2011 a questionnaire was distributed to all of the Trust's junior doctors and SNAs who were involved in out of hours handover to try to further understand the dissatisfaction with the process. The questionnaire was designed using input from junior doctors and SNAs. Questionnaires were distributed by the authors in paper and electronic form. The response rate was $62.5 \%(35 / 56)$ from $31 / 48$ junior doctors and $4 / 8$ SNAs who were spread across all 3 sites. The majority of respondents $(71 \%)$ were not aware of a handover protocol that was in place (and at this time there was no protocol). $69 \%$ of staff had been involved in incidences in the preceding 6 months where handovers had not occurred whilst oncall. Respondents were asked to rate the handover process at their respective hospital site (Scale 1-10, 1=poor, 10=excellent); the overall median score was $5 / 10$. Respondents gave a number of suggestions for improving the process (see Results Table).

See supplementary file: ds1913.doc - "Results Table"

\section{Design}

The project team consisted of a number of junior doctors, SNAs and the Trust's Leadership and Development Tutor. Using the results and suggestions from the December 2011 questionnaire and from an initial project meeting the team designed a handover protocol.
This protocol consisted of guidelines and individual flow-charts for each hospital site, detailing who should handover to whom and at what times. There were different flow charts for handovers on weekdays and for handovers at the weekends. The guidelines supported these flowcharts and explained them more fully. A handover 'jobs folder' was designed to be used at each hospital site. The purpose of this was to have a folder in which details of tasks could be written down to support the verbal handovers taking place. These folders were to be kept in the main offices at each hospital site which were used by the team during their on-calls.

The initial drafts of the protocols, guidelines and jobs folders were shared with a subgroup of junior doctors and SNAs working in BEHMHT. Their feedback was used to further improve these documents before they were implemented across the Trust.

We engaged senior staff (including doctors, managers, human resources, and senior nurses) in the project at the Trust's Medical Education meetings and the monthly 'Mental Health at Night' meetings .

The questionnaire which had been used in December 2011 was used to measure satisfaction with the new handover process. These questionnaires were sent out by the author (JP) in July 2012, at the end of the six month junior doctor rotation, to all junior doctors and SNAs involved in out of hours' handovers. Results were calculated for each site individually and for all three sites combined.

An audit was conducted to assess whether or not out of hours' handovers were taking place at the designated times following the introduction of the guidelines. At each hospital site there was a designated 'junior doctor project representative'. These representatives contacted those involved in each handover at each site to check if the handover had been carried out. If handovers hadn't occurred, the reasons for this were documented. The percentage compliance rate with handovers was calculated for each site. Our standard was that $100 \%$ of handovers should occur, however it was acknowledged that this would be a difficult target to meet given the current difficulties with handover. This standard was chosen because the GMC's 'Good Medical Practice' guidelines state that suitable arrangements should be made for patient care when doctors go off duty. These arrangements should include an 'effective' hand-over; this implies that handover should occur at the end of every shift (15). The audit results were plotted onto runcharts. The results of the questionnaires and audit were analysed using descriptive methods.

\section{Strategy}

First PDSA Cycle (Audit):

In the 'Plan' phase the goals of change were to develop an effective and consistent handover process at BEH-MHT and to improve staff satisfaction with the handover process. In the 'Do' phase the new handover process was implemented on 1/2/12 (when the new rotation of junior doctors started). The junior doctor project representatives gave a presentation at their respective local site inductions on the handover process. This process was discussed 
with each of the SNAs on an individual basis during their supervision sessions with the lead SNA prior to its implementation. Each staff member who was to be involved in the process of out of hours handover was given a copy of the protocol, guidelines, and a sample jobs sheet. Copies of these documents were put up on the walls of the main offices used by the on-call staff.

In the 'Study' phase compliance with handover guidelines was monitored via a daily audit $13 / 2 / 12-26 / 2 / 12$ to assess whether or not handovers were taking place at the designated time slots across the three hospital sites. The results highlighted that there was good compliance with the handover guidelines at Chase Farm Hospital and St Ann's Hospital, but poor compliance with the handover guidelines at the Barnet+Edgware site. The reasons for the poor compliance with the guidelines at this site were examined.

In the 'Act' phase a number of changes were implemented at Barnet+Edgware which included staff being sent email reminders about the importance of handovers. The local site junior doctor project representative gave an informal talk on handovers to the other junior doctors working there. From 5/3/12-1/4/12 staff on-call were contacted on a daily basis to check if handovers had occurred at the appropriate times, if they had not they were reminded to do so in future. Switchboard and Human Resources were contacted to ensure that they had up-to-date rotas and contact details for doctors on-call. It was felt that Chase Farm and Barnet+Edgware sites should adopt the simpler St Ann's model for on-calls which had fewer doctors on-call in a 24 hour period and thus fewer handovers. Unfortunately it was not possible to implement this change at this point in time as the six month junior doctor rota had already been prepared.

\section{Second PDSA Cycle (Audit and Survey):}

The 'Plan' stage of the second PDSA cycle had the same goals of change as the first cycle. The changes which were described in the 'Act' stage of the first cycle were implemented and compliance with the guidelines was monitored in the 'Do' stage. This was done via an audit which was completed every 8 days at all three sites from $5 / 3 / 12-27 / 7 / 12$. Staff satisfaction was tested via a feedback questionnaire in July 2012 at the end of the 6 month junior doctor rotation.

In the 'Study' phase it was noted that there was an improvement at the Barnet+Edgware site with regards to proportion of handovers taking place. The questionnaires highlighted that there was an improvement in staff satisfaction with the handover process compared with the December 2011 questionnaire (baseline measurement).

In the 'Act' phase, to improve the process further, it was felt that the simpler on-call system which was used at St Ann's (where there is one doctor on-call for the 24 hour shift during weekdays rather than three) should be adopted Trust-wide. We approached the Trust's Directors who agreed to this new rota. It was then put through a consultation process and implemented for the new rotation of junior doctors starting in August 2012.
A number of changes were incorporated into the guidelines as a result of feedback received from the questionnaires. A copy of the handover guidelines was put in the induction handbook which each new junior doctor to the Trust receives a copy of. Information on the handover process was given to staff at the Trust's induction in August 2012 and was put on the agenda for future junior doctor inductions. Handovers would continue to be an agenda item at the Trust's regular 'Mental Health at Night' meetings which were attended by junior doctor representatives, the lead SNA, human resources staff, clinical directors and managers.

\section{Post-Measurement}

The results from the daily audit in the first PDSA cycle, 13/2/12-26/2/12, showed that at St Ann's and Chase Farm $100 \%$ of handovers occurred. At Barnet+ Edgware however, only $74 \%$ of audited handovers occurred. The factors surrounding this poor result were multiple and looked into individually.

St Ann's has one duty doctor (9am-9am) and one SNA (9pm-9am) on-call in the 24 hour period where as at Barnet+Edgware there are three doctors (covering the 9-1 pm, 1-5pm, 5pm-9am shifts) and one SNA. We saw that having an increased number of doctors on-call at Barnet+Edgware led to increased confusion for those involved in oncalls as well as for switchboard. This complex on-call system at Barnet+Edgware meant that four handovers (at 9am, 1pm, 5pm and $9 \mathrm{pm}$ ) were required in a 24 hour period compared with only two (9am and 9pm) at St Ann's. Chase Farm has a similar system to Barnet+Edgware with three duty doctors on call and thus four handovers in a 24 hour period, however handovers here appeared to occur more consistently. We hypothesised that this was because of the bleep system used at Chase Farm which necessitates those on-call having to meet face to face to hand the bleep over. There is no bleep system in place at Barnet+Edgware, as is preferred for 'Mental Health at Night', and as a result handovers often take place over the telephone.

Beyond these differences in structure, it was noted that at Barnet+Edgware there were difficulties with switchboard obtaining and using up-to-date rotas and contact details for staff. This led to many attempted handovers via switchboard not occurring. It appeared that some of the Barnet+Edgware junior doctors had not fully understood the new handover process when it was introduced at their local induction day, and it appeared that some doctors were resistant to this new change.

The audit in the second PDSA cycle was completed every 8 days at all three sites from $5 / 3 / 12-27 / 7 / 12$ following the improvements which were made in the first PDSA cycle. The results showed that again at Chase Farm and St Ann's 100\% of handovers occurred. At Barnet+Edgware an improvement was seen as a total of $91 \%$ of their handovers took place. It is evident from the run-chart that there was actually $100 \%$ adherence from the $6 / 4 / 12$ onwards at Barnet+Edgware which coincided with the end of the intensive one month period where staff were contacted on a daily basis about handovers (See runcharts).

The questionnaire in July 2012, which was part of the second PDSA 


\section{BMJ Quality Improvement Reports}

cycle, received a response rate of $67 \%(37 / 55)$ from $7 / 8$ SNAs and $30 / 47$ junior doctors who were spread across the three sites. Some of the SNAs who responded worked at Chase Farm as well as at Barnet+Edgware when on-call, and so they were given their own separate category in the results table. The results showed an overall improvement in satisfaction with handovers compared with the December 2011 questionnaire. 100\% of respondents were aware of the Trust's handover protocol. $35 \%$ of respondents had been involved in incidences on call where handovers had not taken place (the majority were from Barnet and Edgware sites). This was an improvement compared with the December 2011 questionnaire where $69 \%$ of respondents had been involved in such incidences. Respondents scored the handover process at their respective hospital sites as 7/10 (median) showing an improvement compared with the median December 2011 questionnaire score of 5/10 (See Results table).

See supplementary file: ds1914.doc - "Runcharts BMJ"

\section{Lessons and Limitations}

A lesson learnt through the Handover Project was the importance of engaging all stakeholders from the beginning. The project involved large numbers of staff from different disciplines which could have had the potential to lead to difficulties in implementation. Another lesson learnt was the effectiveness of 'bottom-up' change. The guidelines were designed and developed by the junior doctors and SNAs who were actually involved in out of hours' handovers, thus making them appropriate and relevant.

Sustainability has been a key factor in this project. In order to ensure that the progress made by so far is maintained multiple steps have been taken; for example putting the guidelines in the Junior Doctor Handbook and highlighting handovers at the Trust's inductions. Involving senior staff (consultants, lead SNA, managers and human resources) in the project should also help to ensure longevity given that junior doctors regularly rotate placements.

Limitations

A limitation to this project was the lack of baseline audit data which would have been useful to compare our subsequent audits to, in order to demonstrate a change. Another limitation was that the audit only looked at whether or not handovers had taken place, it did not assess the quality of the handovers or assess if the improved handover process affected patient outcomes. It is also worth noting that despite St Ann's and Chase Farm scoring $100 \%$ on their audit, 2 staff members from each site reported in the July 2012 questionnaire that they had been involved in incidences where handovers had not occurred. This highlights one of the difficulties in using the audit method which will not always capture the day-to-day variability in the data. Using the audit and the questionnaire together helped to minimise this variability as adherence to the handover guidelines was captured in both.

There may have been a response bias in our data collection as we could hypothesise that those people who responded to the questionnaires would be more motivated to engage in the handover process. There may have been a specific bias from Barnet+Edgware as we could hypothesise that those doctors and SNAs who were contacted during the intensive monitoring period might be more likely to engage in the handover process.

The December questionnaire result for St Ann's was not representative as the response rate was low at 56\% (9/16) which makes the St Ann's results less valid. It was also noted that the SNA response rate was low at $50 \%(4 / 8)$ and therefore not representative. The questionnaire results were not analysed individually for SNAs and doctors so it is difficult to say how this impacted on the results. There were no clear reasons identified for these varying response rates.

It is worth considering that the new handover process has been largely influenced by external factors such as the introduction of the 'Mental Health at Night' system.

\section{Conclusion}

Overall, the results showed that handovers across the Trust occurred relatively consistently and that staff satisfaction with handovers improved following implementation of the guidelines.

In August 2012, a number of changes were made as highlighted in the 'Act' section of the second PDSA cycle. A third PDSA cycle will monitor these changes via an audit and repeat questionnaire over the next 6 month period.

As a result of the Handover Project, the Trust secured funding from the London Deanery SteLI Initiative (2) to construct a training package on handovers. This was put together by a multiprofessional team under the direction of an external facilitator. There were two pilot training days run in March 2012 which received positive feedback. This training package is now available on the 'Synapse' website (16) for use across the London Deanery. The Trust has more recently secured funding from NHS London and the London Deanery to construct an E-Learning Module on Handovers. This E-learning module will help to improve the quality of handovers and also help to enable the long term sustainability of the Handover Project.

BEH-MHT has developed its own communication tool, specific to mental health, namely SBAR-MH (Situation, Background, Assessment, Recommendation-Mental Health) (17) which is based on the widely used SBAR tool (18). This tool details what information should be considered when giving a handover in a mental healthcare setting. The Trust's Handover Guidelines and $\mathrm{E}$ Learning module will help to link the use of this tool in handovers to better improve communication.

\section{References}

1. General Medical Council, Survey Report - Trainee Survey: All trainees by post specialty by Local Education Provider (2011). Barnet, Enfield and Haringey Mental Health NHS Trust. Available from URL: http://gmc-onlineeducationreport 
s.org/IndicatorScores.aspx?agg=AGG46\%7c2011\&groupclu ster $=4 \% 7 c R R P($ Accessed 1/9/12)

2. London Deanery and NHS London, Simulation and Technology-enhanced Learning Initiative (SteLi). Available from URL: http://www.simulation.londondeanery.ac.uk (Accessed 1/8/12)

3. World Health Organisation, Joint Commission. Communication during patient handovers. Patient saf sol. 2007;1:1-4

4. British Medical Association, National Patient Safety Agency, NHS Modernisation Agency. Safe handover: safe patients. 2005

5. Raduma-Tomàs M, Flin R, Yule S, Williams D. Doctors' handovers in hospital: a literature review. BMJ Qual Saf $2011 ; 20: 128-133$

6. Kitch B, Cooper J, Zapol W, Marder J, Karson A, Hutter M, Campbell E. Handoffs causing patient harm: a survey of medical and surgical house staff. Jt Comm J Qual Patient Saf. 2008 Oct; 34(10):563-70.

7. Cohen M, Hilligoss P. The published literature on handoffs in hospitals: deficiencies identified in an extensive review.

Qual Saf Health Care. 2010; 19: 493-497.

8. Horwitz L, Moin T, Krumholz H, Wang L, Bradley E. Consequences of Inadequate Sign-out for Patient Care. Arch Intern Med. 2008;168(16):1755-1760

9. Petersen L, Brennan T, O'Neil A, Cook A, Lee T. Does housestaff discontinuity of care increase the risk for preventable adverse events? Ann Intern Med. 1994; 121 : 866-872

10. Jagsi R, Kitch B, Weinstein D, Campbell E, Hutter M, Weissman J. Residents report on adverse events and their causes. Arch Intern Med. 2005; 165: 2607-2613

11. Kachalla A, Gandhi T, Puopolo A, Yoon C, Thomas E, Griffey R, Brennan T, Studdert D. Missed and delayed diagnoses in the emergency department: A study of closed malpractice claims from 4 liability insurers. Ann Emerg Med. 2007;49(2):196-205

12. Cleary M, Walter G, Horsfall J. Handover in psychiatric settings: is change needed? J Psychosoc Nurs Ment Health Serv. 2009; 47(3):28-33

13. Yonge $O$. Shift report: a ritual play on a residential adolescent unit. J Psychiatr Ment Health Nurs. 2008; 15(1): 45-51

14. Hunt G, Marsden R, Connor N. Clinical handover in acute psychiatric and community mental health settings. $J$ Psychiatr Ment Health Nurs. 2012; 19(4):310-318

15. General Medical Council, Guidance on Maintaining Good Medical Practice. Available from URL: http://www.gmc-uk.or g/guidance/good medical_practice/working with colleague s_arranging_cover.asp (Accessed 23/8/12)

16. Synapse (London Deanery and NHS London), Interprofessional training package for Handover. 2012. Available from URL:

https://secure.synapse.nhs.uk/groups/simulation-and-techno logy-enhanced-learning-

initiative--steli---/documents/show/4150 (Accessed 1/10/12)

17. Professor Ikkos, Dr Jaydeokar, Dr J Perry, Dr H Omar, Dr R Manghnani, Barbara Bradbury. Barnet, Enfield and Haringey
Mental Health Trust. SBAR-MH (Situation, Background, Assessment, Recommendation- Mental Health), 2012

18. Dr M Leonard et al. SBAR (Situation, Background, Assessment, Recommendation).Kaiser Permanente, Colorado, USA. Taken from the NHS Institute for Innovation and Improvement website. Available from URL:

http://www.institute.nhs.uk/quality_and_service_improvement_tools/ quality and service improvement tools/sbar - situation - backgr ound - assessment - recommendation.html (Accessed 1/8/12)

\section{Declaration of interests}

Nothing to declare

\section{Acknowledgements}

Patient Support Groups from BEH which include Barnet Voice for Mental Health, Haringey User Network and Enfield Mental Health Users Group.

BEH-MHT Staff:

Dr Susie Lingwood, Consultant Psychiatrist, Leadership and Service Improvement Tutor

Dr Sujeet Jaydeokar, Consultant Psychiatrist, STeLI Tutor

Gerard Comey, Lead SNA

Dr Marc Lester, Consultant Psychiatrist, Mental Health at Night Lead Consultant

Suleman Bhana, Joan Francis-Ibrahim Postgraduate Department

Isabelle Iny, Workforce Development

Luis Ferrinho, Human Resources

Dr Liz Sampson, Consultant Psychiatrist, UCL Senior clinical lecturer

Other Doctors and SNAs

Others:

Dr Hussein Omar, SpR, Camden and Islington NHS Trust

Patient Groups from BEH-MHT

Barbara Bradbury, Director of Halland Solutions 